\title{
Prognostic Significance of Regional Arterial Stiffness for Stroke in Hypertension
}

\author{
Takuro Kubozono Mitsuru Ohishi \\ Department of Cardiovascular Medicine and Hypertension, Graduate School of Medical and \\ Dental Sciences, Kagoshima University, Kagoshima, Japan
}

\section{Key Words}

Arterial stiffness - Brachial-ankle pulse wave velocity - Cardio-ankle vascular index .

Carotid-femoral pulse wave velocity

\begin{abstract}
Background: Hypertension is strongly associated with cardiovascular disease. It has been reported that arterial stiffness is related to cardiovascular mortality and morbidity in hypertensive patients and that the physiological evaluation of arterial stiffness may assist clinicians in the early detection of atherosclerosis. Summary: It has been demonstrated that increased arterial stiffness is an independent predictor of cardiovascular disease, including stroke. Arterial stiffness is associated with structural changes in the brain. However, the stiffness responses of muscular arteries are different from those of elastic arteries, and so the impact of arterial stiffness and the conclusions to be drawn may be different depending on the region in which the measurement is taken. Key Messages: In this review, we summarize the current literature describing the association between arterial stiffness, including carotid-femoral and brachial-ankle pulse wave velocity and cardio-ankle vascular index, and cardiovascular disease, specifically stroke. We discuss the utility and prognostic significance of regional arterial stiffness measurements.

(C) 2015 S. Karger AG, Basel
\end{abstract}

\section{Introduction}

Pathological changes in the large arteries make an important contribution to the pathogenesis of cardiovascular disease. The etiology and progression of such arterial changes involve both structural and functional components, yet the details remain poorly understood. Increased arterial stiffness is associated with aging [1], hypertension [2, 3], and end-stage 
renal disease [4-6], and it is a strong predictor of the risk of development of cardiovascular disease, both in patients with hypertension as well as in the general population [7-8].

Pulse wave velocity (PWV) has conventionally been used as a measurement of arterial stiffness, and increased PWV is associated with atherosclerosis. Increased PWV has also been associated with increased risk of cardiovascular disease, including clinical stroke. Specifically, carotid-femoral PWV (cfPWV) is the most well-recognized and established index of arterial stiffness. Recently, additional methods, including brachial-ankle PWV (baPWV) and cardio-ankle vascular index (CAVI), have been developed to evaluate arterial stiffness. The procedure for taking a baPWV measurement is simpler than that required for cfPWV, as it involves the use of an automated device and it does not require exposure of the femoral site. baPWV measurements are highly reproducible, and the values from cfPWV and baPWV are closely related [9-10]. However, it is a problem that both cfPWV and baPWV are affected by changes in blood pressure (BP) during measurement [10]. In contrast, CAVI is adjusted for BP based on the stiffness parameter $\beta$, and it remains a useful index of arterial stiffness independent of BP changes [11-13].

PWV can be determined simultaneously across regional arterial segments, either central or peripheral. Central segments in which PWV is measured are the heart-carotid, heartbrachial, heart-femoral, heart-ankle, and carotid-brachial segments. The peripheral segments are the brachial-ankle and femoral-ankle segments [14]. Central arteries become stiffer during aging because of a decrease in the ratio of elastin to collagen [15-17]. Peripheral arteries, which contain a greater proportion of smooth muscle cells, may not be similarly affected by aging [18]. Although arterial stiffness of different regions is expected to have distinct effects on cardiovascular disease, the clinical impact of regional PWVs on cardiovascular prognosis has not yet been fully investigated.

In this review, we describe the association between arterial stiffness, including cfPWV, baPWV, and CAVI, and cardiovascular disease, specifically stroke. In addition, we discuss the potential prognostic significance of regional arterial stiffness.

\section{Stroke and Arterial Stiffness}

\section{Stroke and cfPWV}

PWV measures the speed of the BP wave as it travels between two different sites within the artery. cfPWV is considered to be the 'gold standard' measurement of arterial stiffness. It allows direct investigation of the association between arterial stiffness and small vessel disease [19]. In order to measure PWV, the arterial path length must be estimated using distance measurements made on the surface of the body. Such sophisticated and complex techniques are inconvenient, which is particularly problematic in large clinical trials.

\section{Measurement of cfPWV}

To measure cfPWV, an automatic device records the time delay $(\mathrm{t})$ between the rapid upstroke of the feet of simultaneously recorded waveforms, which are obtained transcutaneously over the right common carotid artery and the right femoral artery. The distance (D) traveled by these pulse waves are estimated by measuring the distance between the two recording sites over the surface of the body using a tape measure. Aortic PWV is calculated automatically as $\mathrm{D} / \mathrm{t}(\mathrm{m} / \mathrm{s})$. Aortic PWV is widely considered to be the most established index of arterial stiffness [20]. However, there is no standardization or consensus regarding how to measure the arterial path length required for PWV. Sugawara et al. [21] reported that PWV values that account for carotid arterial length were 16-31\% lower than the corresponding PWV calculated using the straight distance between the carotid and femoral sites. 


\section{Utility of cfPWV}

Several reports have noted that increased cfPWV is associated with cerebral small vessel disease. Poels et al. [22] found that arterial stiffness is associated with white matter lesions, independently of other cardiovascular risk factors. In this study, 1,460 participants underwent cfPWV measurements and brain MRI scans. Higher cfPWV was associated with a larger volume of white matter lesions, but not with lacunar infarcts or with microbleeds. In patients with uncontrolled hypertension, a higher cfPWV was significantly associated with a larger volume of white matter lesions, with deep or infratentorial microbleeds, and, to a lesser extent, with lacunar infarcts. Such associations were not observed in patients with controlled hypertension or in patients without hypertension.

Henskens et al. [23] reported that aortic PWV shows a positive association with the extent of white matter hyperintensities and with the presence of silent lacunar infarcts in hypertensive patients who have no history of cardiovascular and cerebrovascular disease, and that this association is independent of age, mean arterial pressure, and other vascular risk factors. The study observed independent associations between cfPWV and manifestations of silent cerebral small vessel disease. Tuttolomondo et al. [24] found that ischemic stroke and metabolic syndrome patients exhibit greater arterial stiffness in relation to age, gender, and cardiovascular risk factors compared to control subjects without a history of stroke, and also compared to stroke patients without metabolic syndrome.

\section{Stroke and baPWV}

Although conventional techniques for the measurement cfPWV are noninvasive, it requires the use of a femoral artery transducer that has been carefully adjusted to obtain an accurate pulse wave, which increases psychologic stress. In contrast, a simple and noninvasive method for automatic measurement of the baPWV has been described. Although some amount of the baPWV value may be determined by peripheral arterial stiffness, the baPWV has been shown to be a valid and reproducible method, closely correlated with cfPWV $[9,10]$.

\section{Measurement of baPWV}

To measure $b a P W V[9,10]$, cuffs were applied to the extremities, and electrocardiographic electrodes were attached to the upper arm. For phonocardiography, a microphone was placed at the second intercostal space at the left margin of the sternum. After the subjects rested in a supine position for $5 \mathrm{~min}$, measurements were taken using a Colin Waveform Analyzer. First, the BP in the extremities was measured by oscillometry. Then, the pulse volume record was taken at a cuff pressure of $60 \mathrm{~mm} \mathrm{Hg}$. The brachial PWV (heart to brachial), right PWV (right upper arm to right ankle), and left PWV (right upper arm to left ankle) were automatically calculated according to the following equations: brachial PWV $=\mathrm{D} 1 / \mathrm{t} 1$, right $\mathrm{PWV}=(\mathrm{D} 2-\mathrm{D} 1) / \mathrm{t} 2$, and left PWV $=(\mathrm{D} 2-\mathrm{D} 1) / \mathrm{t} 3$, where D1 is the distance from the heart to the right upper arm, and D2 is the distance from the heart to the ankle. These distances are calculated automatically, based on the height of the subject. $\mathrm{t} 1$ is the time from the second heart sound recorded on the phonocardiogram to the notch of the pulse volume record taken at the right upper arm. $\mathrm{t} 2$ is the time from the onset of the rise in the pulse volume record taken at the right upper arm to the onset of the rise in the pulse volume record taken at the right ankle. $\mathrm{t} 3$ is the time from the onset of the rise in the pulse volume record taken at the right upper arm to the onset of the rise in the pulse volume record taken at the left ankle.

\section{Utility of baPWV}

Kim et al. [25] reported that baPWV was associated specifically with the presence and burden of intracranial cerebral atherosclerosis in stroke patients, but was not found to be associated with extracranial cerebral atherosclerosis. This study observed a tendency for 
patients with both intracranial and extracranial cerebral artery atherosclerosis to have higher baPWV than those patients with only intracranial cerebral artery atherosclerosis. The authors speculated that age, which is associated with baPWV, might contribute to a higher baPWV in patients with both intracranial and extracranial cerebral artery atherosclerosis. They also noted that it is possible that patients with both intracranial and extracranial cerebral artery atherosclerosis were already at a more advanced stage of cerebral artery atherosclerosis and that they may have progressed to more severe atherosclerosis in the distal intracranial cerebral arteries. The report concluded that their findings may suggest that increased arterial stiffness promotes atherosclerosis in the distal intracranial cerebral artery that supplies the end organ. Measurement of baPWV may serve as a useful tool for the selection of patients who have an elevated risk of developing intracranial cerebral atherosclerosis.

Several reports have addressed the issue of an appropriate baPWV cutoff value for the prediction of cardiovascular events. Tomiyama et al. [26] reported that baPWV of $1,700 \mathrm{~cm} / \mathrm{s}$ is predictive of posthospitalization cardiovascular events, and $1,800 \mathrm{~cm} / \mathrm{s}$ is predictive of major cardiovascular events. It has been reported that $1,750 \mathrm{~cm} / \mathrm{s}$ is an appropriate baPWV cutoff value for predicting the onset of stroke, cardiovascular disease, cardiovascular disease plus stroke, and mortality in hypertensive patients [27].

Elevated cfPWV is associated with both higher risk and higher associated mortality of cardiovascular events, such as coronary heart disease and stroke. Recently, Kim et al. [28] reported that baPWV serves as a strong predictor with respect to mortality of patients with acute stroke. They evaluated the prognostic value of baPWV in 1,765 patients who had been admitted for acute ischemic stroke and who had completed measurement of baPWV during the admission process. During a mean follow-up period of $3.33 \pm 1.57$ years, there were 228 deaths by all causes, including 143 vascular deaths. In a multivariate Cox hazard regression analysis, patients in the highest tertile of baPWV values $(>2,263 \mathrm{~cm} / \mathrm{s})$ exhibited an increased risk both of death by all causes and of vascular death in particular, compared with patients in the lowest tertile $(<1,779 \mathrm{~cm} / \mathrm{s})$.

\section{Stroke and CAVI}

The major limitation of baPWV is that the measurement is affected by changes in BP during measurement. To address this shortcoming, CAVI has been developed as a novel atherosclerotic index that incorporates PWV and BP measurements. CAVI is adjusted for BP based on the stiffness parameter $\beta$, and it is therefore expected to measure arterial stiffness independent of BP. We found that both baPWV and CAVI demonstrated positive correlations with age and with systolic BP, but that CAVI showed a weaker correlation with systolic BP compared to baPWV. Although the baPWV value was shown to increase in proportion to the corresponding increase in systolic BP, the CAVI value did not show a significant change between the two measurements [13].

\section{Measurement of CAVI}

CAVI is measured using a Vasera instrument (Fukuda Denshi, Tokyo, Japan) [11-13]. To take the measurement, cuffs were applied to the four extremities of the subject, and electrocardiographic electrodes were attached to the upper arm. A microphone was placed on the sternal angle for phonocardiography. The subject then rested in the supine position for $5 \mathrm{~min}$. The PWV was calculated by dividing the distance from the subjects' aortic valve to their ankle artery by the sum of the difference between the time the pulse waves were transmitted to the brachium and the time the same wave was detected at the ankle, and the time difference between the second heart sound on the phonocardiogram and the notch of the brachial pulse wave $[11,12]$. To minimize the effect of cuff inflation on blood flow dynamics, pulse waves were measured with cuffs that were inflated to less than diastolic BP (50 $\mathrm{mm} \mathrm{Hg}$ ), and 
extremity BP was measured by oscillometry. The systolic and diastolic BP and the pulse pressure were obtained by measurements taken at the right brachial artery.

To obtain the CAVI, the stiffness parameter $\beta$ was substituted into the equation for determining vascular elasticity and PWV, as described below. The stiffness parameter $\beta$ indicates patient-specific, BP-independent vascular stiffness as measured by arterial ultrasound [29] and is calculated as follows:

Stiffness parameter $\beta=[\operatorname{In}(\mathrm{Ps} / \mathrm{Pd})] \times(\mathrm{D}+\Delta \mathrm{D}) / \Delta \mathrm{D}$

where Ps and Pd are the systolic and diastolic BP in $\mathrm{mm} \mathrm{Hg}$, respectively. D is the diameter of the blood vessel and $\Delta \mathrm{D}$ is the change in $\mathrm{D}$.

PWV can then be estimated by the Bramwell-Hill equation as follows:

$(\mathrm{D}+\Delta \mathrm{D}) / \Delta \mathrm{D}=2 \rho \times 1 /(\mathrm{Ps}-\mathrm{Pd}) \times \mathrm{PWV}^{2}$

where $\rho$ is the density of blood.

If we substitute equation 2 into equation 1 , we obtain the following:

Stiffness parameter $\beta=2 \rho \times 1 /(\mathrm{Ps}-\mathrm{Pd}) \times \operatorname{In}(\mathrm{Ps} / \mathrm{Pd}) \times \mathrm{PWV}{ }^{2}=\mathrm{CAVI}$.

Utility of CAVI

Several studies have examined the relationship between stroke and CAVI. Suzuki et al. [30] reported that CAVI is significantly higher in patients with ischemic cerebrovascular diseases than in healthy control subjects, particularly in those patients with white matter ischemic lesions, large artery atherosclerosis, and small vessel occlusion. However, no difference in CAVI was observed between patients with transient ischemic attack and control subjects.

In another study, cerebral small vessel disease was found to be significantly associated with CAVI after adjustment for pulse pressure and systolic BP [31]. In this report, silent cerebral small vessel diseases were observed in $4.1 \%$ of the participants, even including asymptomatic young and middle-aged subjects (mean age of $50 \pm 7$ years). Subjects with small vessel diseases tended to be older and to have higher systolic BP and higher CAVI than those without small vessel diseases. CAVI showed a stepwise incremental increase in value between the hypertensive subjects without and those with small vessel disease. The authors also showed that, in contrast to CAVI, pulse pressure, which is an indirect measurement of arterial stiffness, was not significantly different between subjects with and without small vessel disease.

In addition, Saji et al. [32] reported that silent brain infarction is associated with arterial stiffness as measured by CAVI in elderly patients (mean age of 69 years). The CAVI cutoff value determined for the indication of silent brain infarct was 9.2, and the value for white matter hyperintensities was 8.9. Patients with CAVI $\geq 9.2$ had a higher odds ratio with respect to the presence of both silent brain infarct and white matter hyperintensities, compared with patients with CAVI $<9.2$, after adjustment for age and gender.

\section{Differential Impact of Regional PWVs}

Muscular arteries, such as the femoral or brachial arteries, show different stiffness responses than elastic arteries, such as the aorta [16]. Whereas the central arteries become stiffer with aging due to a decrease in the ratio of elastin to collagen [15-17], the peripheral arteries, which contain a greater proportion of smooth muscle cells, may not be similarly affected by aging [18]. PWVs can be measured between multiple atrial sites, and there are several reports about the differential usefulness of these regional PWVs. 
Kubozono and Ohishi: Prognostic Significance of Regional Arterial Stiffness for Stroke in Hypertension

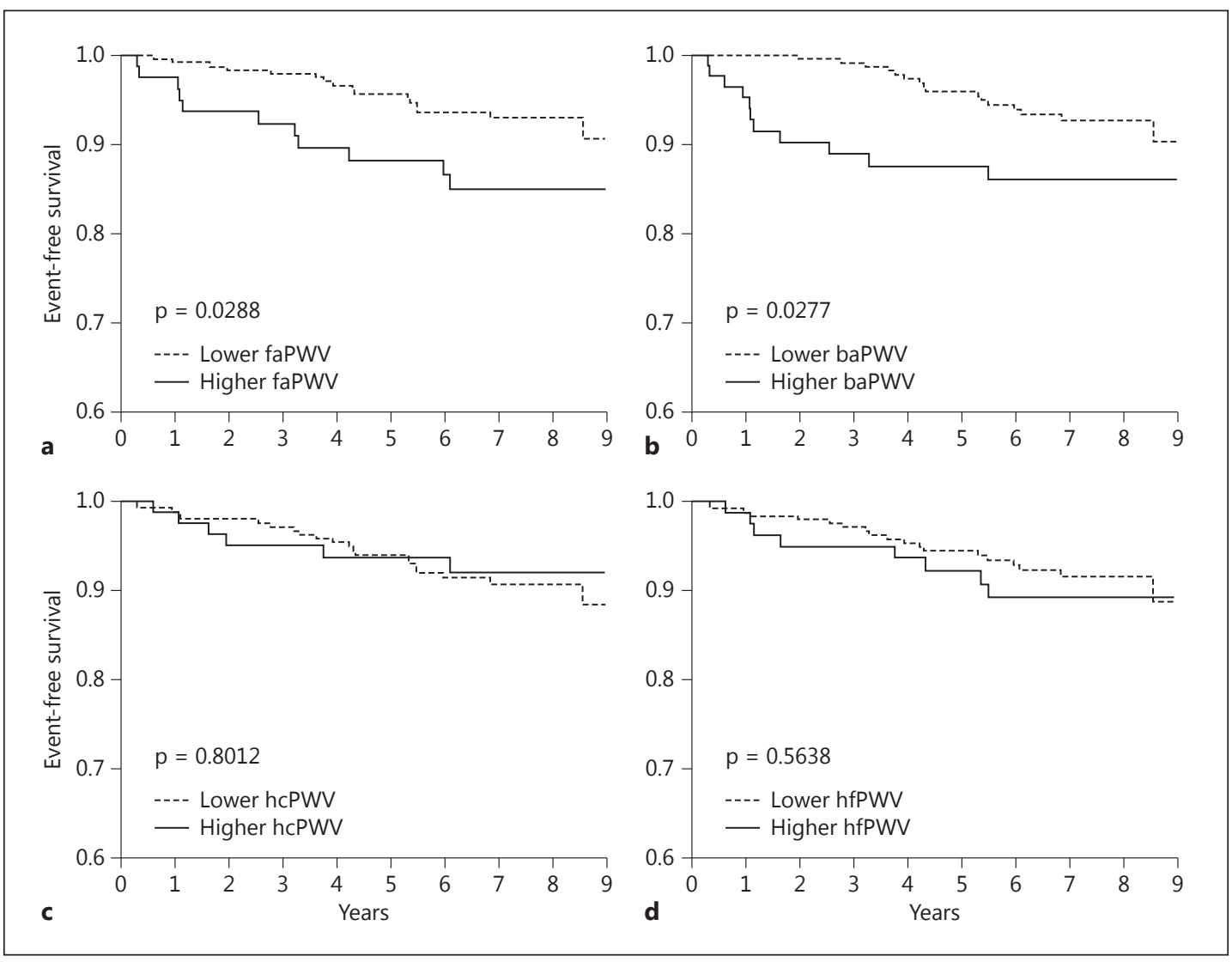

Fig. 1. a-d The association between regional PWVs and stroke. Subjects with higher femoral-ankle PWV (faPWV) and baPWV had a significantly higher incidence of stroke. hcPWV = Heart-carotid PWV; hfPWV = heart-femoral PWV.

In healthy subjects, cfPWV exhibits a significant and strong correlation with central heart-femoral PWV, whereas baPWV exhibits a significant and moderate correlation with both central heart-femoral PWV and peripheral femoral-ankle PWV [33]. In patients with type 2 diabetes mellitus, Hatsuda et al. [34] measured regional PWV in 4 segments of arteries to examine whether ischemic heart disease is more closely associated with the stiffness of central arteries than the stiffness of peripheral arteries, and their findings indicated that central arterial stiffness plays an important role in the development of ischemic heart disease. They also found that the degree of increased arterial stiffness associated with chronic kidney disease in type 2 diabetes patients varies among arterial regions, and that decreased glomerular filtration rate had the strongest impact on the stiffness of the aorta among the 4 regions that were examined [35].

Kawai et al. [36] examined the association between regional PWVs and cardiovascular outcomes. In this study, Kaplan-Meier analysis showed no statistically significant difference in mortality between subjects with high and low femoral-ankle PWV, although subjects with higher baPWV, heart-carotid PWV, and heart-femoral PWV exhibited a nonsignificant trend toward higher mortality. On the other hand, the study found that subjects with higher femoralankle PWV and baPWV had a significantly higher incidence of stroke (fig. 1). They concluded that central arterial stiffness had a stronger clinical impact on total mortality than peripheral arterial stiffness, and that peripheral arterial stiffness had a stronger clinical impact on the incidence of stroke than central arterial stiffness. 
Kubozono and Ohishi: Prognostic Significance of Regional Arterial Stiffness for Stroke in Hypertension

\section{Summary}

Arterial stiffness is predictive of cardiovascular events and mortality by all causes in both hypertensive patients and in the population at large. Herein we have reviewed the literature regarding the utility of regional arterial stiffness and found that the prognostic impact of regional PWV is still unclear. Further studies are needed to evaluate the usefulness of regional PWV.

\section{References}

1 Asmar R: Factors influencing pulse wave velocity; in Asmar R: Arterial Stiffness and Pulse Wave Velocity Clinical Applications. Paris, Elsevier Science Publishing 1999, pp 57-63.

-2 Safar ME, Frohlich ED: The arterial system in hypertension: a prospective view. Hypertension 1995;26:10-14.

-3 Shimizu Y, Itoh T, Hougaku H, Nagai Y, Hashimoto H, Sakaguchi M, Handa N, Kitagawa K, Matsumoto M, Hori M: Clinical usefulness of duplex ultrasonography for the assessment of renal arteriosclerosis in essential hypertensive patients. Hypertens Res 2001;24:13-17.

$\checkmark 4$ Kawada H, Sumimoto T, Okayama H, Hiwada K: Structure and function of the left ventricle and carotid artery in hemodialysis patients. Hypertens Res 2001;24:221-227.

-5 Rahn KH, Barenbrock M, Hausberg M, Kosch M, Suwelack B, Witta J: Vessel wall alterations in patients with renal failure. Hypertens Res 2000;23:3-6.

-6 Narita I, Okada M, Omori S, Nagai M, Sawanaka N, Kondo D, Goto S, Shimada H, Shimotori T, Arakawa M, Gejyo F: The circadian blood pressure rhythm in non-diabetic hemodialysis patients. Hypertens Res 2001;24:111117.

7 Boutouyrie P, Tropeano AI, Asmar R, Gautier I, Benetos A, Lacolley P, Laurent S: Aortic stiffness is an independent predictor of primary coronary events in hypertensive patients: a longitudinal study. Hypertension 2002;39:10-15.

8 Blacher J, Asmar R, Djane S, London GM, Safar ME: Aortic pulse wave velocity as a marker of cardiovascular risk in hypertensive patients. Hypertension 1999;33:1111-1117.

-9 Yamashina A, Tomiyama H, Takeda K, Tsuda H, Arai T, Hirose K, Koji Y, Hori S, Yamamoto Y: Validity, reproducibility, and clinical significance of noninvasive brachial-ankle pulse wave velocity measurement. Hypertens Res 2002;25:359-364.

10 Kubo T, Miyata M, Minagoe S, Setoyama S, Maruyama I, Tei C: A simple oscillometric technique for determining new indices of arterial distensibility. Hypertens Res 2002;25:351-358.

11 Yambe T, Yoshizawa M, Saijo Y, Yamaguchi T, Shibata M, Konno S, Nitta S, Kuwayama T: Brachio-ankle pulse wave velocity and cardio-ankle vascular index (CAVI). Biomed Pharmacother 2004;58(suppl 1):S95-S98.

12 Shirai K, Utino J, Otsuka K, Takata M: A novel blood pressure-independent arterial wall stiffness parameter; cardio-ankle vascular index (CAVI). J Atheroscler Thromb 2006;13:101-107.

13 Kubozono T, Miyata M, Ueyama K, Nagaki A, Otsuji Y, Kusano K, Kubozono O, Tei C: Clinical significance and reproducibility of new arterial distensibility index. Circ J 2007;71:89-94.

14 Kim WJ, Park CY, Park SE, Rhee EJ, Lee WY, Oh KW, Park SW, Kim SW, Song S: The association between regional arterial stiffness and diabetic retinopathy in type 2 diabetes. Atherosclerosis 2012;225:237-241.

15 Cameron JD, Bulpitt CJ, Pinto ES, Rajkumar C: The aging of elastic and muscular arteries: a comparison of diabetic and nondiabetic subjects. Diabetes Care 2003;26:2133-2138.

16 van der Heijden-Spek JJ, Staessen JA, Fagard RH, Hoeks AP, Boudier HA, van Bortel LM: Effect of age on brachial artery wall properties differs from the aorta and is gender dependent: a population study. Hypertension 2000; 35:637-642.

17 Mitchell GF, Parise H, Benjamin EJ, Larson MG, Keyes MJ, Vita JA, Vasan RS, Levy D: Changes in arterial stiffness and wave reflection with advancing age in healthy men and women: the Framingham Heart Study. Hypertension 2004; 43:1239-1245.

18 Greenwald SE: Ageing of the conduit arteries. J Pathol 2007;211:157-172.

19 Laurent S, Cockcroft J, Van Bortel L, Boutouyrie P, Giannattasio C, Hayoz D, Pannier B, Vlachopoulos C, Wilkinson I, Struijker-Boudier H; European Network for Non-invasive Investigation of Large Arteries: Expert consensus document on arterial stiffness: methodological issues and clinical applications. Eur Heart J 2006; 27:2588-2605.

20 Safar ME, London GM: Therapeutic studies and arterial stiffness in hypertension: recommendations of the European Society of Hypertension. The Clinical Committee of Arterial Structure and Function. Working Group on Vascular Structure and Function of the European Society of Hypertension. J Hypertens 2000;18:15271535.

21 Sugawara J, Hayashi K, Yokoi T, Tanaka H: Carotid-femoral pulse wave velocity: impact of different arterial path length measurements. Artery Res 2010;4:27-31. 
Kubozono and Ohishi: Prognostic Significance of Regional Arterial Stiffness for Stroke in Hypertension

22 Poels MM, Zaccai K, Verwoert GC, Vernooij MW, Hofman A, van der Lugt A, Witteman JC, Breteler MM, MattaceRaso FU, Ikram MA: Arterial stiffness and cerebral small vessel disease: the Rotterdam Scan Study. Stroke 2012;43:2637-2642.

23 Henskens LH, Kroon AA, van Oostenbrugge RJ, Gronenschild EH, Fuss-Lejeune MM, Hofman PA, Lodder J, de Leeuw PW: Increased aortic pulse wave velocity is associated with silent cerebral small-vessel disease in hypertensive patients. Hypertension 2008;52:1120-1126.

24 Tuttolomondo A, Di Raimondo D, Di Sciacca R, Pecoraro R, Arnao V, Buttà C, Licata G, Pinto A: Arterial stiffness and ischemic stroke in subjects with and without metabolic syndrome. Atherosclerosis 2012;225:216-219.

25 Kim J, Cha MJ, Lee DH, Lee HS, Nam CM, Nam HS, Kim YD, Heo JH: The association between cerebral atherosclerosis and arterial stiffness in acute ischemic stroke. Atherosclerosis 2011;219:887-891.

-26 Tomiyama H, Koji Y, Yambe M, Shiina K, Motobe K, Yamada J, Shido N, Tanaka N, Chikamori T, Yamashina A: Brachial-ankle pulse wave velocity is a simple and independent predictor of prognosis in patients with acute coronary syndrome. Circ J 2005;69:815-822.

27 Kawai T, Ohishi M, Onishi M, Ito N, Takeya Y, Maekawa Y, Rakugi H: Cut-off value of brachial-ankle pulse wave velocity to predict cardiovascular disease in hypertensive patients: a cohort study. J Atheroscler Thromb 2013;20:391-400.

28 Kim J, Song TJ, Song D, Lee KJ, Kim EH, Lee HS, Nam CM, Nam HS, Kim YD, Heo JH: Brachial-ankle pulse wave velocity is a strong predictor for mortality in patients with acute stroke. Hypertension 2014;64:240-246.

29 Hirai T, Sasayama S, Kawasaki T, Yagi S: Stiffness of systemic arteries in patients with myocardial infarction: a noninvasive method to predict severity of coronary atherosclerosis. Circulation 1989;80:78-86.

30 Suzuki J, Sakakibara R, Tomaru T, Tateno F, Kishi M, Ogawa E, Kurosu T, Shirai K: Stroke and cardio-ankle vascular stiffness index. J Stroke Cerebrovasc Dis 2013;22:171-175.

-31 Choi SY, Park HE, Seo H, Kim M, Cho SH, Oh BH: Arterial stiffness using cardio-ankle vascular index reflects cerebral small vessel disease in healthy young and middle aged subjects. J Atheroscler Thromb 2013;20:178185.

-32 Saji N, Kimura K, Shimizu H, Kita Y: Silent brain infarct is independently associated with arterial stiffness indicated by cardio-anklevascular index (CAVI). Hypertens Res 2012;35:756-760.

33 Choo J, Shin C, Barinas-Mitchell E, Masaki K, Willcox BJ, Seto TB, Ueshima H, Lee S, Miura K, Venkitachalam L, Mackey RH, Evans RW, Kuller LH, Sutton-Tyrrell K, Sekikawa A: Regional pulse wave velocities and their cardiovascular risk factors among healthy middle-aged men: a cross-sectional population-based study. BMC Cardiovasc Disord 2014;14:5.

-34 Hatsuda S, Shoji T, Shinohara K, Kimoto E, Mori K, Fukumoto S, Koyama H, Emoto M, Nishizawa Y: Regional arterial stiffness associated with ischemic heart disease in type 2 diabetes mellitus. J Atheroscler Thromb 2006;13:114-121.

-35 Kimoto E, Shoji T, Shinohara K, Hatsuda S, Mori K, Fukumoto S, Koyama H, Emoto M, Okuno Y, Nishizawa Y: Regional arterial stiffness in patients with type 2 diabetes and chronic kidney disease. J Am Soc Nephrol 2006; 17:2245-2252.

36 Kawai T, Ohishi M, Onishi M, Ito N, Takeya Y, Oguro R, Takami Y, Yamamoto K, Rakugi H: Prognostic impact of regional arterial stiffness in hypertensive patients. Heart Vessels 2014, Epub ahead of print. 